\title{
MATLAB analysis and simulink model for amplitude modulation technique
}

\author{
G.N Kareem *, G.A Gbotoso and S.O Omogoye \\ Department of Electrical and Electronics Engineering, School of Engineering, Lagos State Polytechnic, Ikorodu, Lagos, \\ Nigeria.
}

World Journal of Advanced Engineering Technology and Sciences, 2021, 02(02), 021-028

Publication history: Received on 27 March 2021; revised on 02 May 2021; accepted on 04 May 2021

Article DOI: https://doi.org/10.30574/wjaets.2021.2.2.0035

\begin{abstract}
Communication plays an indispensable part in several facets of the human existence. To communicate or transmit information over a long distance, modulation is required. This paper presents amplitude modulation (A.M) in which the amplitude of the carrier is altered in accordance to the input (message) signal, while we keep the frequency constant. The modulated signal (carrier) contains the spectral components which is in the frequency domain analysis. The key concept and principle of A.M are presented with several illustrations with MATLAB and Simulink model to make it easier to understand.
\end{abstract}

Keywords: Amplitude modulation; Modulation; Modulation factor; MATLAB

\section{Introduction}

During the last two decades, the world has witnessed rapid evolution of mobile communication technologies with the help of modulation. Generation cellular system differ, in general, in four main aspects: access to radio network, rate of data transmission, bandwidth and switching schemes. The techniques for transmission of information from 1G (First Generation) system, mainly analogue system, up to the present generation, 5th generation (5G) which is based on digital transmission cannot be achieved without modulation. Frequency modulation (FM) is the method of transmitting information on a carrier wave by changing its frequency. This method is widely used in telecom devices to transmit voice without disruptions. In applications involving analogue operation, the carrier's instantaneous frequency and the instantaneous input signal are directly proportional to each other. Digital information transmission can be achieved using a technique known as frequency-shift keying which involves shifting the carrier frequency among a lot of discrete values, but in this report, we want to look at amplitude modulation (AM) on how it is being modelled at the transmitter end using MATLAB and Simulink model. MATLAB enables manipulation of matrix elements, function and data tracking, implementation of algorithm, creation of user interface and it can be interfaced with other programs in other languages [1]. Simulink which is interactive and a graphical tool was added to MATLAB allow for modelling and simulation of various systems with the ease of connecting predefined and designed building blocks.

\section{Related works}

This paper includes a modulation simulator which is made by using MATLAB GUI. It should however be noted that Modulation is the process of making easier and conveying one or more kind of wave signal to the receiver through the use of a carrier signal, information signal is transmitted [2]. With the help of modulation information can be transmitted to a far distance using carrier signal. The maximum range or distance the information can travel is determined by Friis formula. Constructing frequency modulation radio channels is a very demanding task. In fact, experimentation is too costly and the problems encountered in such designs seem too complicated to be analysed [3] and requires basic

\footnotetext{
* Corresponding author: G.N Kareem

Department of Electrical and Electronics Engineering, School of Engineering, Lagos State Polytechnic, Ikorodu, Lagos, Nigeria. 
knowledge of amplitude modulation. The main criteria for selection of modulation procedure are as follows: power efficiency, spectral efficiency, and system complexity [4]. Energy efficiency of modulation procedure is immediately specified by the required signal/interference ratio, for a given probability of error per bit, BER (Bit Error Rate, BER) in a channel with noise. If we believe the power efficiency of a transition procedure, it can be reasoned that it is neater if the lower signal to noise ratio is necessary for the same probability of error per bit. The energy efficiency criterion is often called a probability of failure of the modulation procedure. The spectral efficiency of the modulation procedure is defined as the ratio of equivalent binary flux, $\mathrm{V}_{\mathrm{b}}$, and frequency bandwidth, Bo, required to transmit the observed signal. This varies according to the type of modulation procedure applied, which are frequency modulation, phase modulation, digital modulation etc.

The complexity of the system is determined by the complexity of the technical implementation of the system and the number of electronic circuits required to complete the system. The price of production will also be considered during the analysis of system complexity. [3] Suggested that simulation of frequency modulation can be achieved using MATLAB and Simulink model and this system modelling and computer simulation of the real physical systems offer a better alternative. Basically amplitude modulation can both be modelled and simulated on a Simulink model and result obtained on a graph of a scope embedded in the software is displayed, also the spectrum analyser if available is used to display the magnitude of the modulated signal against frequency by displaying the sidebands and centre frequencies [5]. Also [6] suggested that message signal can be generated from a pure sine or cosine wave as message signal and the carrier wave can be a square wave which produces carrier pulses can be combined in a PAM (Pulse Amplitude Modulation) modulator circuit to produce pulse amplitude modulated signal. The modulation index or factor $\mathrm{m}$, is defined as the ratio of magnitude or strength of modulating signal to magnitude or strength of carrier signal, where in most cases the magnitude can be measured in volts. Various values of $\mathrm{m}$, dictate the type of modulation in question. For a perfect modulation the value of $m=1$, when the value of $m<1$, then it is under modulation, with the value of $m>1$, then it is over modulation [7].

\section{Mathematical model}

The basic idea behind the model used for the simulation of amplitude modulation is shown in fig.1.

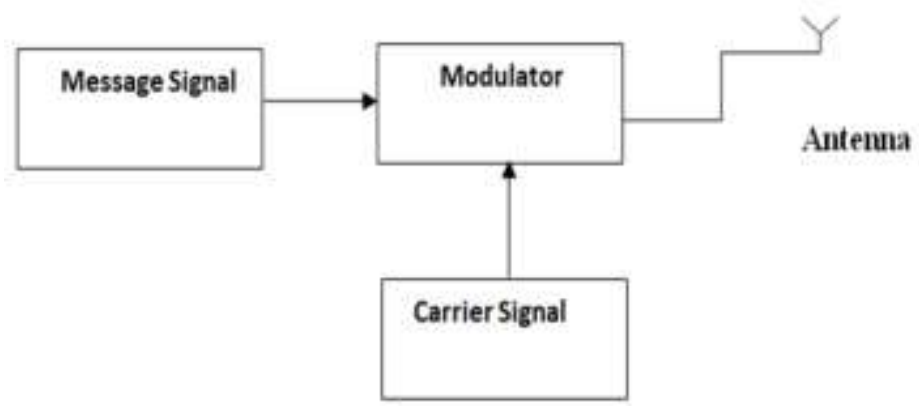

Figure 1 Simple block diagram of signal modulation

From fig. 1 it is observed that the modulator combines the message signal to be transmitted with a carrier signal and the modulated signal is transmitted through the antenna in form of electromagnetic waves. The velocity of the electromagnetic wave, $c$, is related to the wavelength of the wave as:

$$
c=f^{*} \lambda
$$

Where $\mathrm{c}=3 \mathrm{x} 10^{8} \mathrm{~m} / \mathrm{s} \mathrm{f}=$ frequency of transmission, $\lambda=$ wavelength of the wave

\subsection{Representation of the a.m wave in time domain}

The modulating signal is represented by the equation;

$$
Y_{m}=A_{m} * \sin \left(2 * \pi * F_{m} * t\right)
$$

Where $\mathrm{F}_{\mathrm{m}}=$ modulating signal frequency

$$
\mathrm{T}=\text { time and } \mathrm{A}_{\mathrm{m}}=\text { Amplitude of modulating signal }
$$


The carrier signal is represented by the equation:

$$
Y_{c}=A_{c} * \sin \left(2 * \pi * F_{c} * t\right)
$$

The amplitude of the A.M wave is represented by the equation:

$$
Y_{\max }=A_{c}+A_{m} * \sin \left(2 * \pi * F_{m} * t\right)
$$

Where modulation factor, $m=\frac{A_{m}}{A_{c}}$ which implies that:

$$
A_{m}=m A_{C}
$$

Equation (4) becomes:

$$
Y_{\max }=A_{c}\left(1+m * \sin \left(2 * \pi * F_{m} * t\right)\right.
$$

The instantaneous value of Amplitude modulation is represented by the equation:

$$
Y_{\text {ins }}=A_{c}\left(1+m * \sin \left(2 * \pi * F_{m} * t\right) \sin \left(2 * \pi * F_{c} * t\right)\right.
$$

Equation (6) is the amplitude modulated signal equation in time domain implemented in Matlab window. The MATLAB program is shown below.

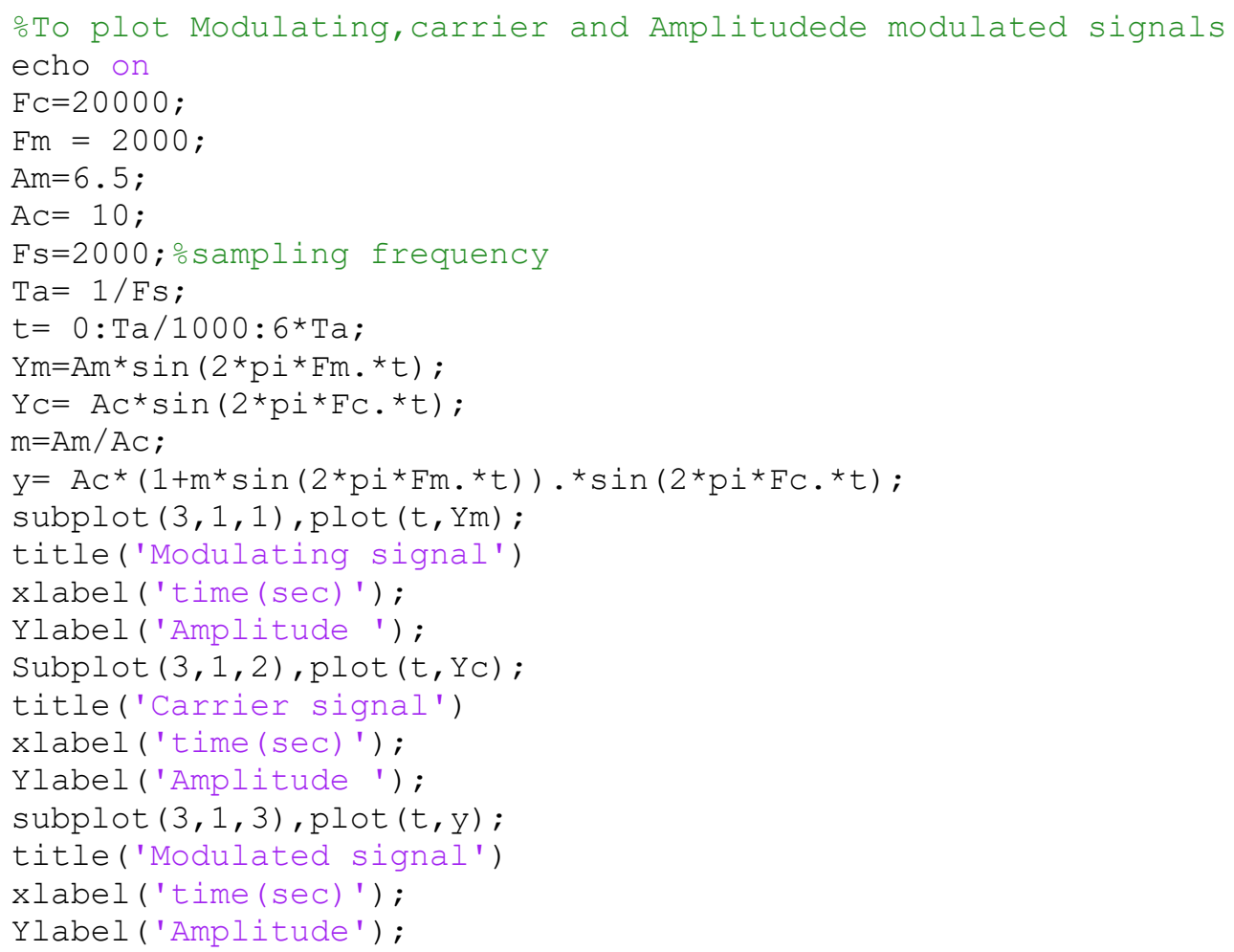

\subsection{Model of the a.m wave equation}

The amplitude modulated signal was modelled using the Simulink software by interconnecting the blocks required to achieve desired result. This is shown in fig. 2 . 


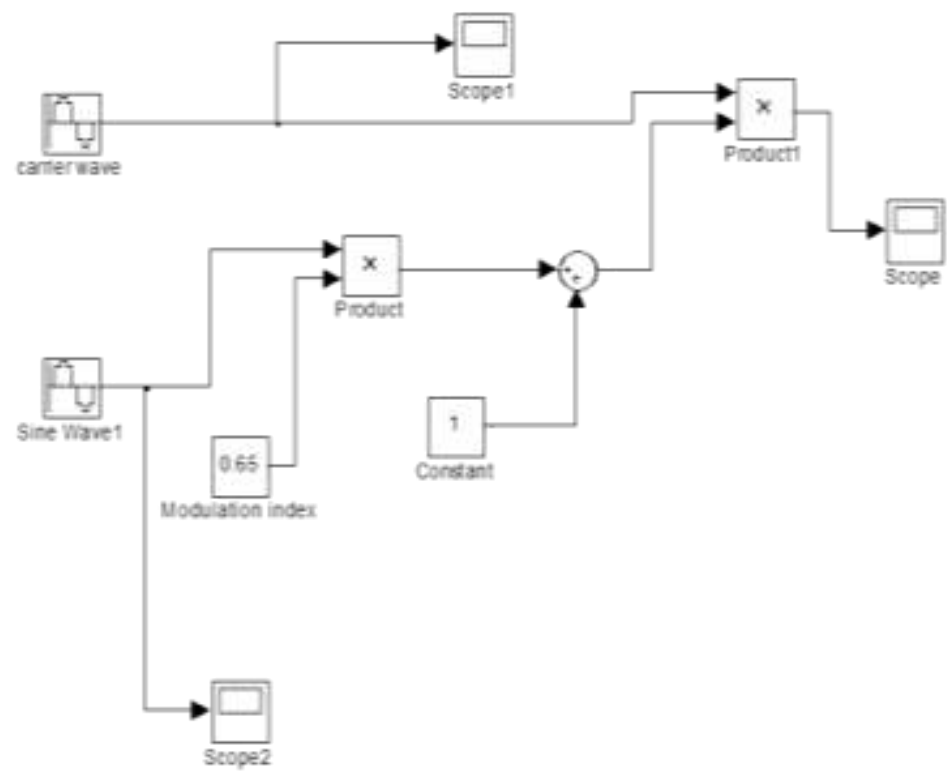

Figure 2 SIMULINK model of Amplitude modulation

The result of the model in figure 2 was achieved by setting the parameters of the modulating signal and carrier signal as shown in fig. 3 and fig. 4 respectively.

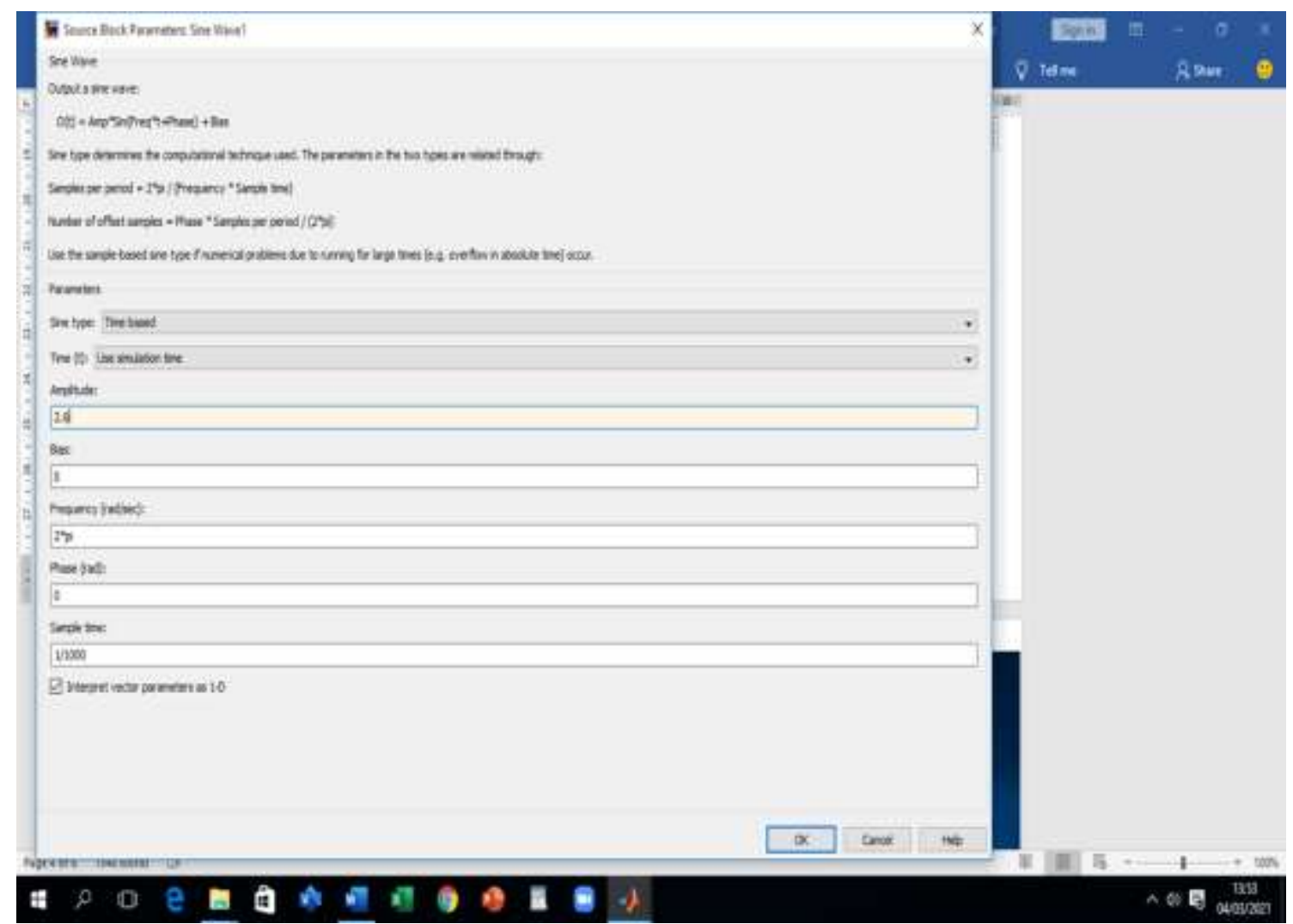

Figure 3 Parameters used for the modulating signal 


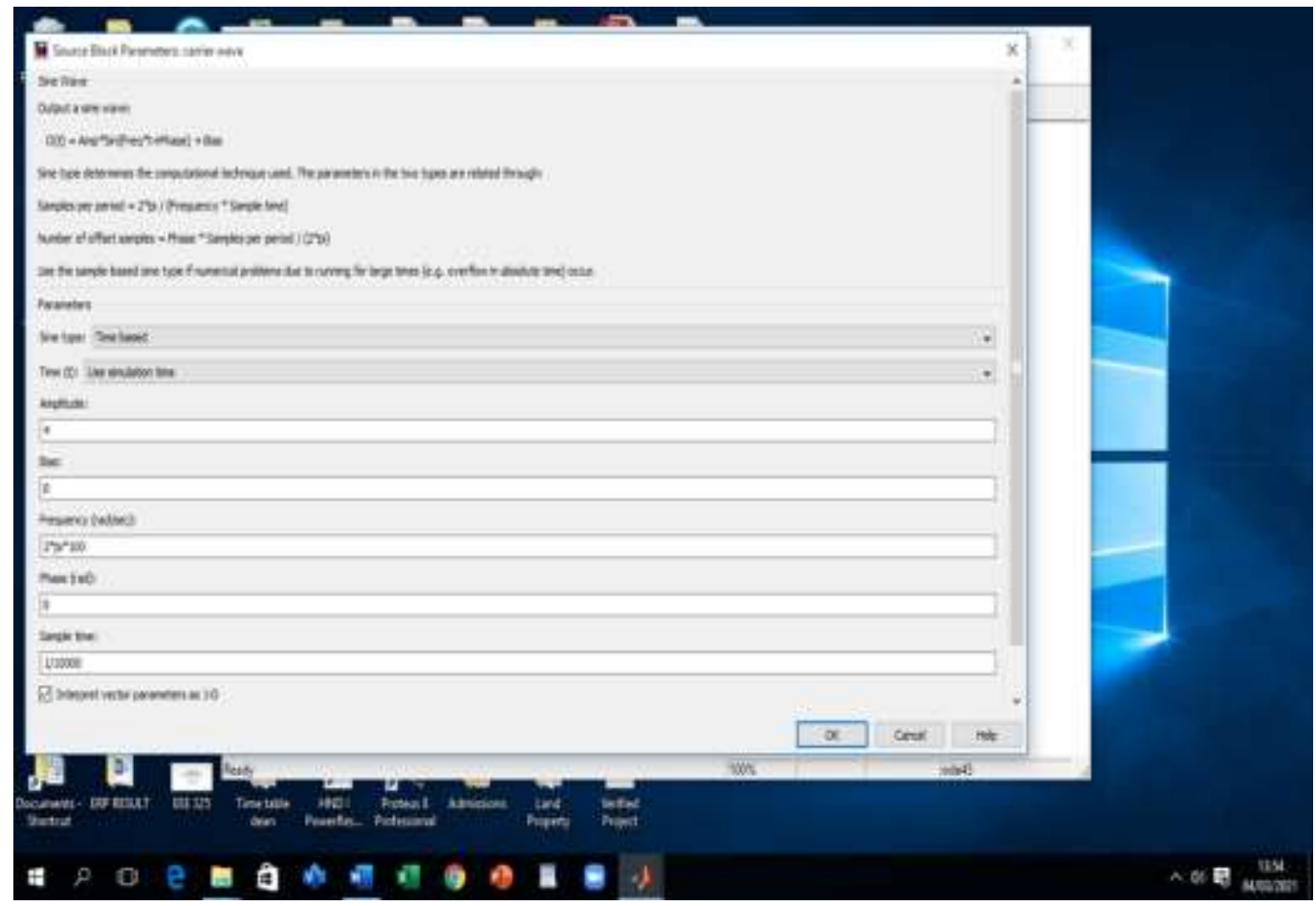

Figure 4 Parameters used for the carrier signal

It will be observed that the amplitude of the modulating and carrier signals were set to 2.6 and 4 respectively to achieve a modulation index of 0.65 which is in conformity with the modulation index block model set for the amplitude modulated signal. It is also observed that the constant block with a value of 1 is added to the product block model which consist of the modulation index and the modulating signal. The combination of which is multiplied in the second product block model with the amplitude of the carrier signal.

\section{Results}

The obtained result is in conformity with the signals expected to be displayed such as the modulating signal, carrier signal and modulated signal with modulation factor of 0.65 is based on the MATLAB program written based on the parameter values set. This is shown in fig. 5,6 and 7 respectively.

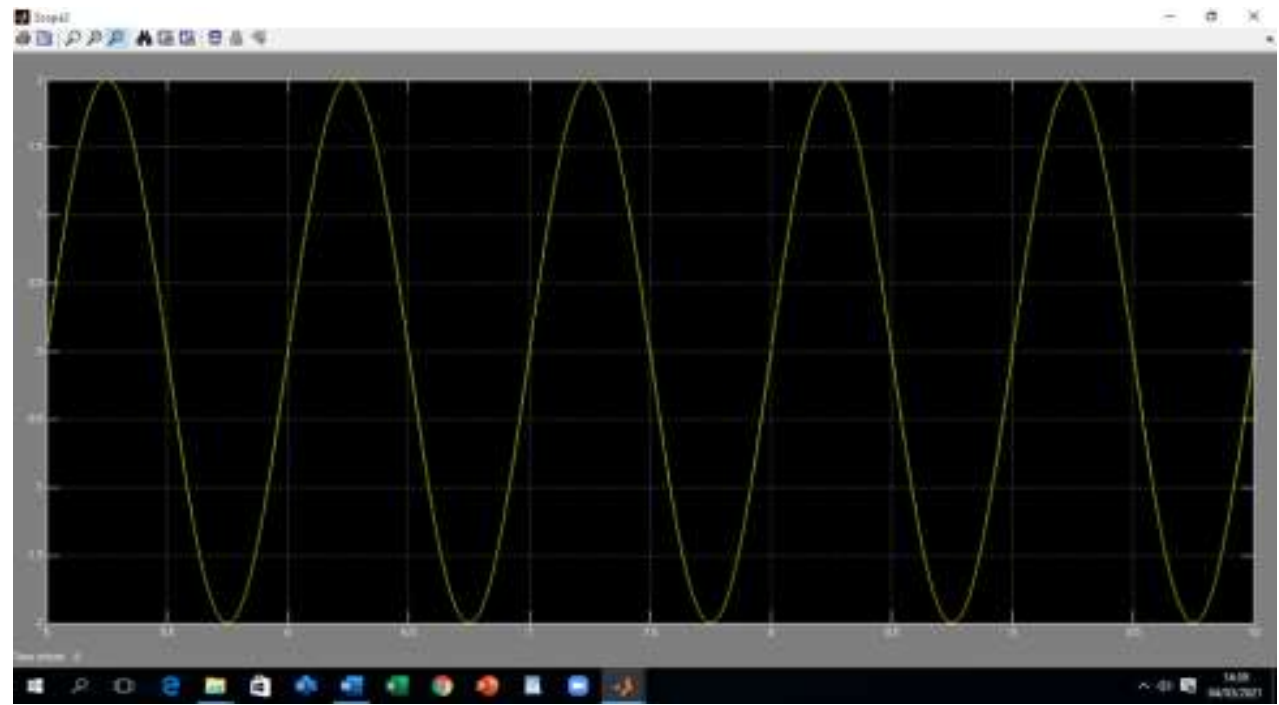

Figure 5 Modulating signal 


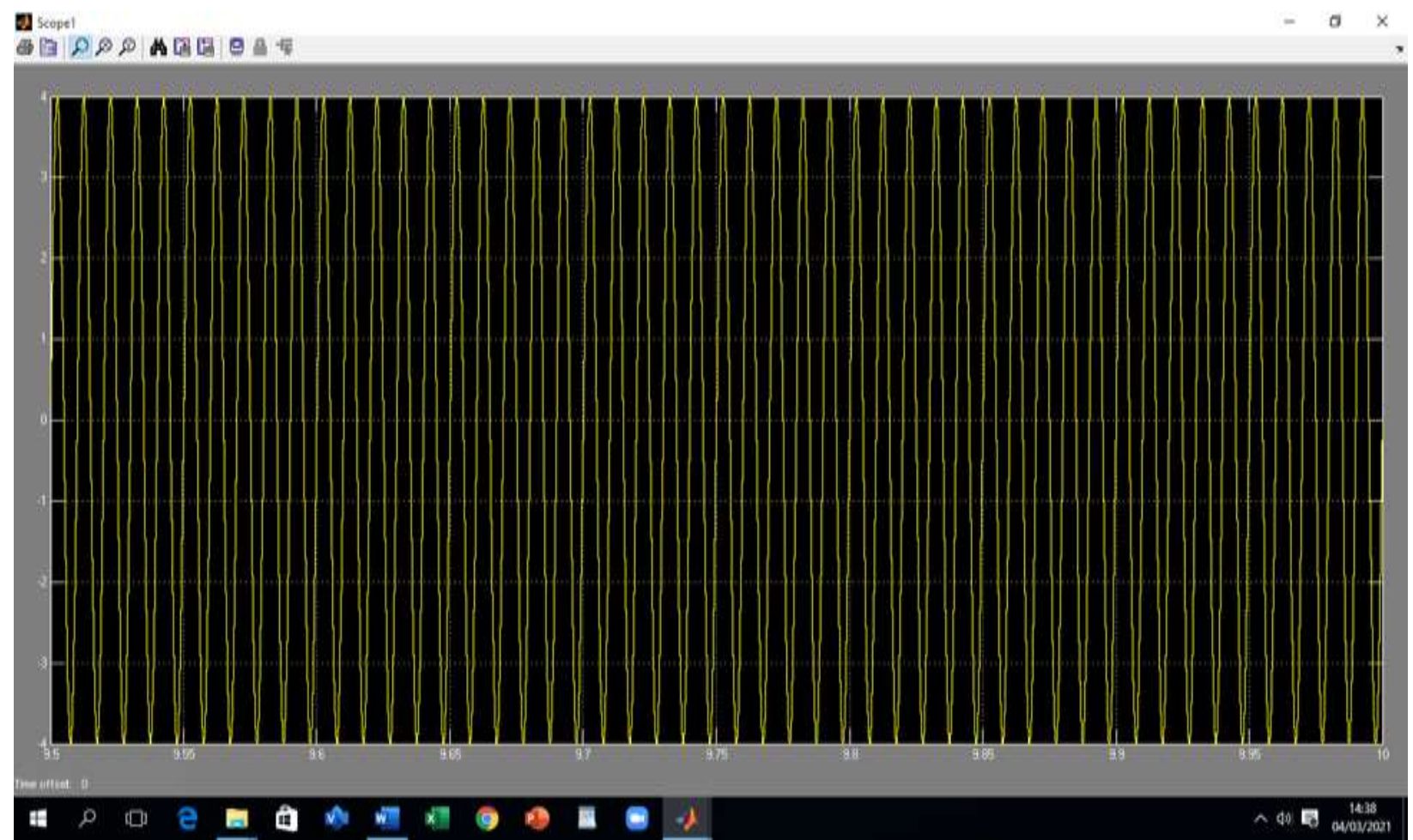

Figure 6 Carrier signal

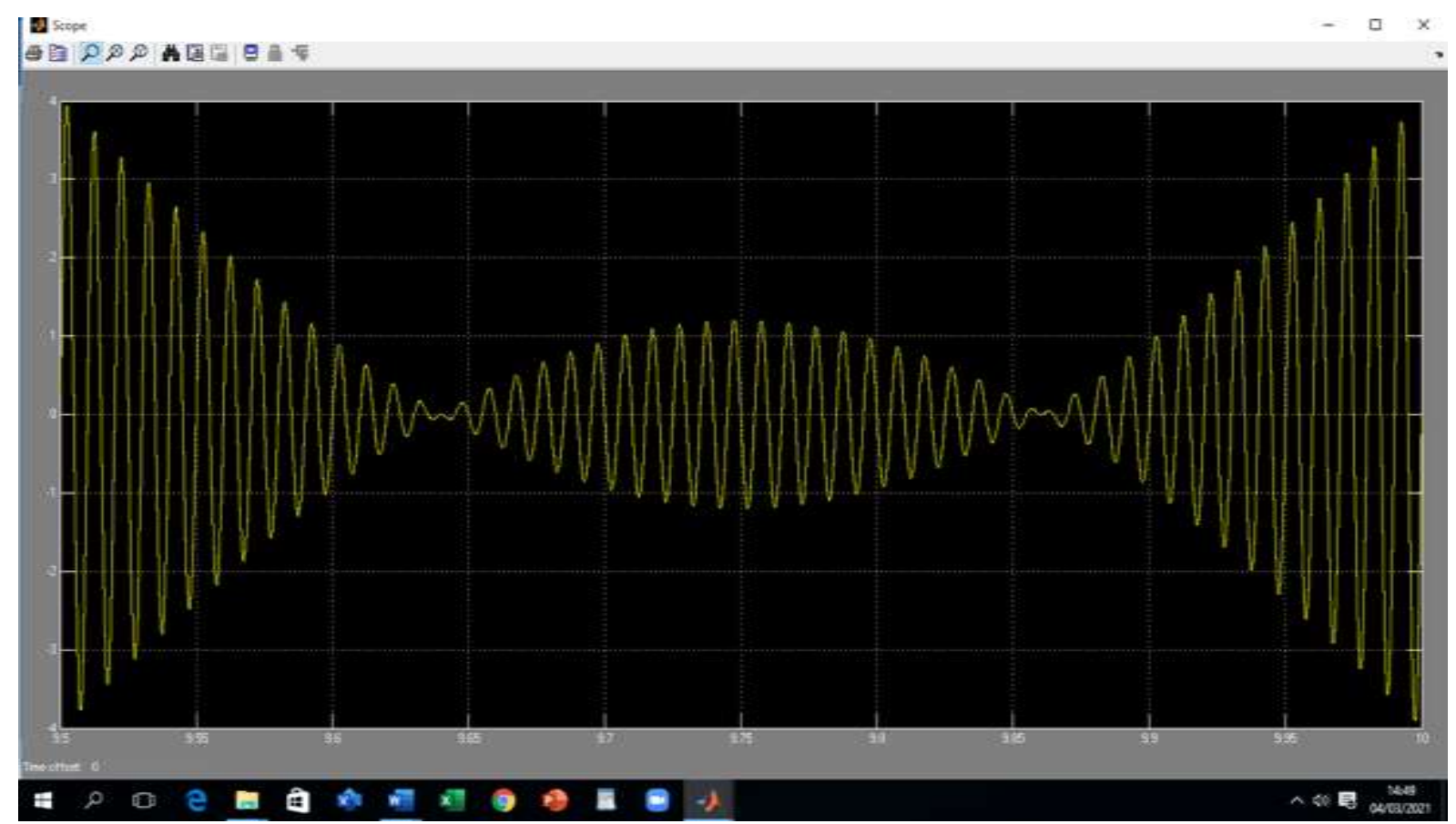

Figure 7 Amplitude Modulated Signal with 0.65 modulation factor

When the modulation factor or index is adjusted to 0.5 by setting the ratio of the amplitude of modulating signal to that of the carrier signal to be $50 \%$,then we have a more perfect modulated signal, which is shown in fig.8 .Overmodulation is shown in fig. 9 .

We can observe that the amplitude modulated signal in figure has a little distortion after each cycle of the modulated signal meaning that it is tending towards overmodulation. 


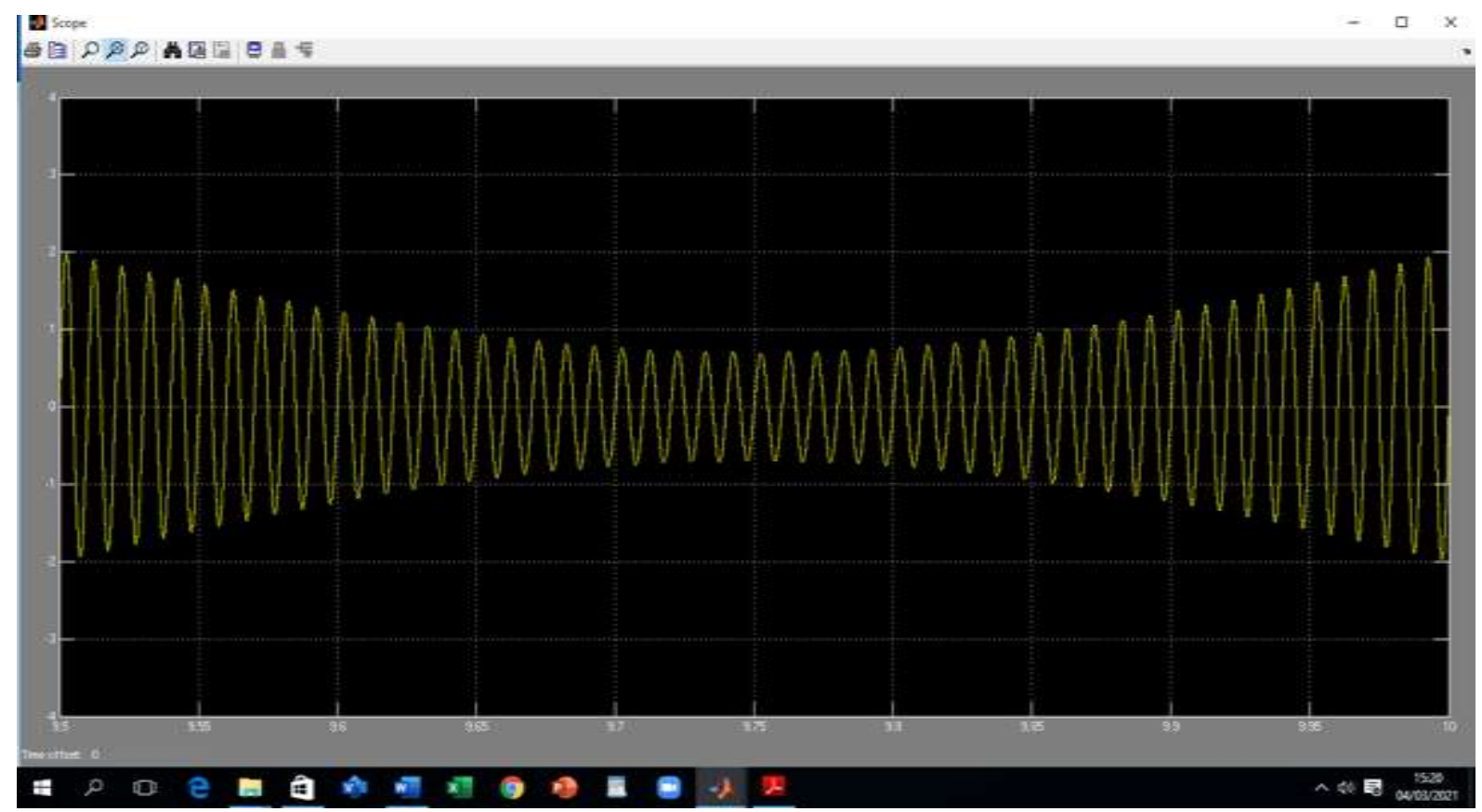

Figure 8 Amplitude Modulated Signal with 0.5 modulation factor

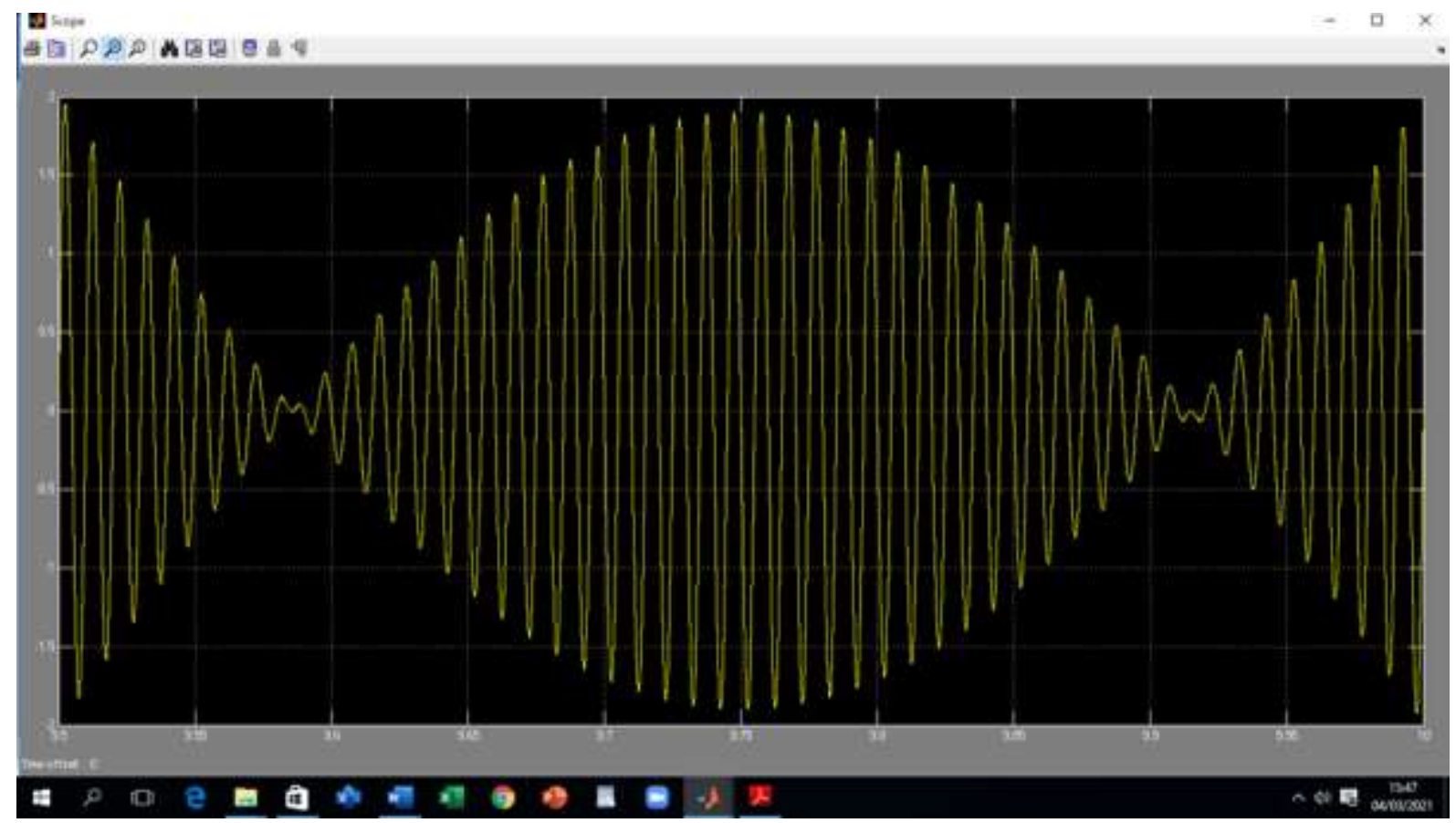

Figure 9 Amplitude Modulated Signal with 1.5 modulation factor

\section{Conclusion}

This paper has been able to implement amplitude modulation with the use of A.M equation implemented in MATLAB environment. Also, the Simulink library tool was used as to model amplitude modulation with the use of communication blocks in the library. We were able to generate the modulating signal, carrier signal and modulated signal. Fig. 9 shows an amplitude modulated signal with modulation factor greater than 1.0, which is called over modulation, (in other word it is not a perfect modulation). It can be concluded that this type of amplitude modulation depends on the modulation 
factor. A modulation factor less than 1.0 is good for transmission of information because there is no distortion(overmodulation).

\section{Compliance with ethical standards}

\section{Acknowledgments}

I acknowledge the God Almighty for the grace he gave me to carry out this research. The authors would like to thank the support of the TETFUND and the Departments of Electrical \& Electronics Engineering, Lagos State Polytechnic.

\section{References}

[1] M Boulmalf, Y Semmar, A Lakas, K Shuaib. Teaching digital and analog modulation to undergradute information technology students using Matlab and Simulink, 2010 IEEE Educ. Eng. Conf. EDUCON. 2010; 685-691.

[2] T Özer, H Çimen, A Akbal. A New Educational Modulation Simulator Using for Digital and Analog Modulations," 2nd Intl' Conf. on Advances in Eng. Sciences and Applied Mathematics (ICAESAM'2014). May 2014; 29-34.

[3] O Lawrence. Modelling and Simulation of Frequency Modulated Radio Channel Using Matlab and Simulink, J. of Multidisciplinary Eng. Science and Tech. Apr.2015; 2(4): 868-872.

[4] D Mitić, A Lebl. Selection procedure for modulation using simulation in Matlab, MIPRO 2011 - 34th Int. Conv. Inf. Commun. Technol. Electron. Microelectron. - Proc. 2011; 547-552.

[5] K Ashlesha, D Harshal, M Kanika et al. Design and Analysis of Amplitude and frequency modulation using matlab simulink," World Journal of Engineering and Rsearch Tech. Aug.2016; 4(2): 302-313.

[6] K Wagh. MATLAB Programming \& Simulink Model for Pulse Amplitude Modulation technique, Int. J. of Electrical, Electronics \& Communication Engineering. Aug 2018; 2(1): 1-8.

[7] R Suryawanshi, VD Patil. Simulink Implementation of Amplitude Modulation Technique using Matlab, Int Research J. of Eng. and Tech. Aug. 2018; 5(8): 185-189. 